\title{
Differential Geometry Based Vector Fields for Characterizing Surrounding Structures of Pulmonary Nodules
}

\author{
Y. Kawata, N. Niki, H. Ohmatsu', M. Kusumoto ${ }^{\text {b }}$, R. Kakinuma ${ }^{a}$,

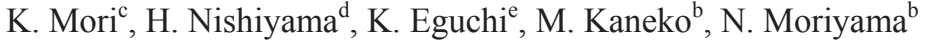 \\ Dept. of Optical Science, Univ. of Tokushima, Tokushima, \\ ${ }^{\mathrm{a}}$ National Cancer Center Hospital East, ${ }^{\mathrm{b}}$ National Cancer Center Hospital, \\ ${ }^{\mathrm{c}}$ Tochigi Cancer Center, ${ }^{\mathrm{d}}$ The Social Health Insurance Medical Center, \\ ${ }^{\mathrm{e} N a t i o n a l ~ S h i k o k u ~ C a n c e r ~ C e n t e r ~ H o s p i t a l ~}$
}

\begin{abstract}
This paper presents a scheme to analyze surrounding structures of pulmonary nodules by using differential geometry based vector fields. In this scheme the differential characteristics such as the principal curvatures and directions are computed from the differential values of the isointensity surfaces. Each voxel in the nodule surrounding is described in terms of shape index and curvedness derived from the principal curvatures. Two vector fields are formed from the directions of the maximum principal curvatures of nodule surrounding and gradient vectors of nodule surface, respectively. The gradient vector field is computed by diffusing the gradient vector on the nodule surface. The regions corresponding to the cylindrical or conic figures which are similar to vessel and plural images are segmented by the shape index and curvedness values. Then, the relationship between the segmented regions and the nodule is evaluated by the inner product of the direction of the maximum principal curvature and the gradient vector. We demonstrate the feasibility of the scheme to classify benign and malignant nodules.
\end{abstract}

\section{Introduction}

Pulmonary nodules remain a common and difficult diagnostic problem. It is often the case that the differential diagnosis by means of transbronchial or percutaneous biopsies becomes difficult due to the nodule size. There has been a considerable amount of interest in the use of thin-section CT images to observe small pulmonary nodules for differential diagnosis without invasive operation [1], [2]. A number of investigators have developed feature extraction and classification methods for characterization of pulmonary nodules. One promising area of recent researches has been the analysis of three-dimensional (3-D) pulmonary nodule images. In assessing the malignant potential of pulmonary nodules, characterizing the relationships between nodules and their surrounding structures such as vessel, bronchi, and pleura is an important step [1], [2]. In order to analyze the relationships several researches concentrate on evaluating the angle between the nodule and line patterns such as vessel and brachia. There are two major approaches to the development of computing directions of line patterns. One approach uses the center line of the line pattern 
obtained by a 3-D thinning process [3], [4]. The direction of the line pattern is approximated by the gradient of the center line. The other approach uses the partial derivatives of 3-D gray level images. The direction of the line pattern is approximated by the eigenvectors of Hessian matrix or the direction of principal curvatures [5], [6]. Although the results of these studies were obtained by small data set or simulations, they indicate the potential of using computerized feature extraction techniques to improve the diagnostic accuracy of differentiating malignant and benign nodules.

In our previous work, we found that curvature indexes such as shape index and curvedness was useful for characterizing the internal structure of nodules [7], [8], [9]. In the present study, we introduce the differential geometrical quantities for the structure analysis of the nodule surrounding and explore the feasibility of differential geometry based vector fields in classifying malignancies from other lesions.

\section{Methods}

\subsection{Extraction of Nodule and Its Surrounding Region}

Thin-section CT images were obtained by the helical CT scanner (Toshiba TCT900S Superhelix). Per patient, about 60 slices at $1 \mathrm{~mm}$ intervals were obtained to observe whole nodule region and its surrounding. The range of pixel size in each square slice of 512 pixels was between $0.3 \times 0.3 \mathrm{~mm}^{2}$ and $0.4 \times 0.4 \mathrm{~mm}^{2}$. The $3 \mathrm{D}$ thoracic image was reconstructed by a linear interpolation technique to make each voxel isotropic.

The segmentation of the 3D pulmonary nodule image consists of three steps [7] ;1)extraction of lung area, 2) selection of the region of interest (ROI) including the nodule region, and 3 ) nodule segmentation based on a geometric approach. The lung area extraction step plays an essential role when the part of a nodule in the peripheral lung area touches the chest wall. The ROI including the nodule was selected interactively. A pulmonary nodule was segmented from the selected ROI image by the geometric approach proposed by Caselles [10]. The region of nodule surrounding was obtained by the subtraction of the segmented 3-D nodule image $\mathrm{I}_{\mathrm{A}}$ from its morphological filtering image $\mathrm{I}_{\mathrm{F}}=\mathrm{I}_{\mathrm{A}}+\mathrm{n}_{\mathrm{B}} \mathrm{I}_{\mathrm{B}}\left(\mathrm{n}_{\mathrm{B}}\right.$ dilations of the image $\mathrm{I}_{\mathrm{A}}$ by structure element $\left.I_{B}\right)$. The structure element used was a $3 \times 3 \times 3$ non-null uniform square centered in the origin.

\subsection{Differential Geometry Based Vector Field}

Each voxel in the region of interest (ROI) including the pulmonary nodule was locally represented by two curvature indexes that represented the shape index and the curvedness. By assuming that each voxel in the ROI lies on the surface which has the normal corresponding to the 3-D gradient at the voxel, we computed directly the curvatures on each voxel from the first and the second derivatives of the gray level image of the ROI by using an approach proposed by Thirion [11]. Let $I(\mathbf{x}): \mathbf{R}^{3} \rightarrow \mathbf{R}$ is a ROI image defined in $\mathbf{R}^{3}$. To compute the partial derivatives of the ROI images $I$ $(\boldsymbol{x})$, the ROI images were blurred by convolving with a 3-D Gaussian function with 
width $\sigma$. The computation of the blurred derivatives were efficiently performed by using the recursive implementation of the Gaussian function [12]. Using the formulas proposed by Thirion [11], Gaussian curvature $K(\boldsymbol{x} ; \sigma)$ and mean curvature $H(\boldsymbol{x} ; \sigma)$ were computed and then the principal curvatures and directions were obtained. The Gaussian and mean curvatures are defined by

$$
\begin{aligned}
& K(\mathbf{x} ; \sigma)= \frac{1}{h^{2}}\left[I_{x}{ }^{2}\left(I_{y y} I_{z z}-I_{y z}{ }^{2}\right)+2 I_{y} I_{z}\left(I_{x z} I_{x y}-I_{x x} I_{y z}\right)\right. \\
&+I_{y}{ }^{2}\left(I_{x x} I_{z z}-I_{x z}{ }^{2}\right)+2 I_{x} I_{z}\left(I_{y z} I_{x y}-I_{y y} I_{x z}\right) \\
&\left.+I_{z}{ }^{2}\left(I_{x x} I_{y y}-I_{x y}{ }^{2}\right)+2 I_{x} I_{y}\left(I_{x z} I_{y z}-I_{z z} I_{x y}\right)\right] \\
& H(\mathbf{x} ; \sigma)=\frac{1}{2 h^{3 / 2}}\left[I_{x}{ }^{2}\left(I_{y y}+I_{z z}\right)-2 I_{y} I_{z} I_{y z}+I_{y}{ }^{2}\left(I_{x x}+I_{z z}\right)-2 I_{x} I_{z} I_{x z}\right. \\
&\left.+I_{z}{ }^{2}\left(I_{x x}+I_{y y}\right)-2 I_{x} I_{y} I_{x y}\right]
\end{aligned}
$$

where $h=I_{x}{ }^{2}+I_{y}{ }^{2}+I_{z}{ }^{2}, \mathrm{x}, \mathrm{y}$, and $\mathrm{z}$ subscripts denote partial derivatives with respect to the corresponding variable. The principal curvatures $\kappa_{1}(x ; \sigma)$ and $\kappa_{2}(x$; $\sigma)\left(\kappa_{1}(x ; \sigma) \geq \kappa_{2}(x ; \sigma)\right)$ are obtained by

$$
\kappa_{i}=H(\mathbf{x} ; \sigma) \pm \sqrt{H^{2}(\mathbf{x} ; \sigma)-K(\mathbf{x} ; \sigma)}(i=1,2) .
$$

The principal directions $\mathbf{v}_{1}$ and $\mathbf{v}_{2}$ are defined by

$$
\mathbf{v}_{i}(\mathbf{x} ; \sigma)=\mathbf{a} \pm \sqrt{H^{2}(\mathbf{x} ; \sigma)-K(\mathbf{x} ; \sigma)} \mathbf{b} \quad(i=1,2) .
$$

where $\mathbf{b}=\left(I_{\mathrm{z}}-I_{\mathrm{y}}, I_{\mathrm{x}}-I_{\mathrm{z}}, I_{\mathrm{y}}-I_{\mathrm{x}}\right)$ and the $\mathrm{x}$ component of the vector $\mathbf{a}$ is obtained by

$$
\begin{aligned}
\mathbf{a} \cdot \mathbf{x}= & -\frac{1}{2 h^{3 / 2}}\left[-2 I_{z}{ }^{3} I_{x y}+I_{y}{ }^{3} I_{z z}+2 I_{y}{ }^{3} I_{x z}-2 I_{y}{ }^{2} I_{z} I_{x y}\right. \\
& +2 I_{z}{ }^{2} I_{x} I_{y z}+2 I_{z}{ }^{2} I_{y} I_{x z}-2 I_{y}{ }^{2} I_{x} I_{y z}-2 I_{z} I_{x} I_{y} I_{z z} \\
& +2 I_{x} I_{y} I_{z} I_{y y}+I_{y}{ }^{2} I_{z} I_{x x}-2 I_{z}{ }^{2} I_{x} I_{x z}+I_{z} I_{x}{ }^{2} I_{z z} \\
& -I_{x}{ }^{2} I_{z} I_{y y}+2 I_{z}{ }^{2} I_{y} I_{y z}-I_{z} I_{y}{ }^{2} I_{z z}+I_{z}{ }^{3} I_{x x}-I_{z}{ }^{3} I_{y y} \\
& -I_{y}{ }^{2} I_{x} I_{x z}+2 I_{x}{ }^{2} I_{y} I_{y z}-I_{y}{ }^{3} I_{x x}+2 I_{x} I_{z}{ }^{2} I_{x y}-I_{y} I_{z}{ }^{2} I_{x x} \\
& -2 I_{z} I_{y}{ }^{2} I_{y z}+I_{y} I_{z}{ }^{2} I_{y y}-2 I_{z} I_{x}{ }^{2} I_{y z}+2 I_{x} I_{y}{ }^{2} I_{x y}+I_{x}{ }^{2} I_{y} I_{z z}-I_{x}{ }^{2} I_{y} I .
\end{aligned}
$$

The $y$ and $z$ components are obtained by circular permutation of $\mathrm{x}, \mathrm{y}$, and $\mathrm{z}$. In tubelike structures such as vessel and pleura, axial directions of them are approximately parallel to the direction of maximum principal curvature of the central part of isointensity surface of the structure. Therefore, a vector field of the nodule surrounding was represented by the direction of the maximum principal curvature. We call this vector field the maximum principal curvature vector ( MPV ) field. 
The original shape index defined by Koendrink [13] gives a continuous distribution of surface types between -1 and 1 . To introduce the shape spectral function of the objects surface patch, Dorai [14] modified the original definition of the shape index so that the shape index maps the surface types on the interval between 0 and 1 . In any definition the distinct surface type corresponds to a unique value of the shape index except the planar surface. Since points on planar surface have an indeterminate shape index, Dorai assigned a symbolic label (a shape index value of 2.0) for the points on planar surface [14]. The definition of the shape index used here was based on the modified definition and [8],[9]. The shape index with the width $\sigma$ at the voxel $\boldsymbol{x}$ was given by

$$
S(\mathbf{x} ; \sigma)=\frac{1}{2}+\frac{1}{\pi} \arctan \frac{\kappa_{1}(\mathbf{x} ; \sigma)+\kappa_{2}(\mathbf{x} ; \sigma)}{\kappa_{1}(\mathbf{x} ; \sigma)-\kappa_{2}(\mathbf{x} ; \sigma)} .
$$

The curvedness quantifies how highly curved a surface is, and is inversely proportional to the size of the object. The curvedness $R(\boldsymbol{x} ; \sigma)$ was given by

$$
R(\mathbf{x} ; \sigma)=\sqrt{\frac{\kappa_{1}(\mathbf{x} ; \sigma)^{2}+\kappa_{2}(\mathbf{x} ; \sigma)^{2}}{2}}
$$

The selection of the width $\sigma$ of the Gaussian function is a critical issue. In previous work [8], [9] when the curvature indexes with width $\sigma$ was utilized to characterize the internal structure of pulmonary nodules, we assigned the value 2.0 to the width, which provided high accuracy of classification between malignant and benign nodules for discrete width values using a data set. In this present study, we also assigned the value 2.0 to the width.

\subsection{Gradient Vector Field}

In order to quantify the relationship between the nodule and its surrounding structure such as vessel and pleura, we focus on two indicators of malignancy, which are denoted as vascular convergence and pleural retraction [1], [2]. In the 3-D thoracic CT images, these findings are observed so that the vessel and pleura images are drawn in the nodule. The shape of the vessel and the pleura images are similar to cylindrical or conic structures. Therefore, we measured an amount of the vascular convergence and pleural retraction by computing the absolute value of the inner product of the directions of cylindrical or conic structures and the normal directions of the nodule surface.

The directions of cylindrical or conic structures were obtained by the MPV field. The normal directions of the nodule surface at the vicinity of the nodule was estimated by the following procedure. This procedure was based on the idea which was proposed by $\mathrm{Xu}$ [15] to improve the segmentation accuracy of the snake model.

Let $g(\mathbf{x}): \mathbf{R}^{3} \rightarrow \mathbf{R}$ is an edge map defined in $\mathbf{R}^{3}$ and $\mathrm{f}(\mathbf{x}): \mathbf{R}^{3} \rightarrow \mathbf{R}$ is a segmented binary image of the nodule defined in $\mathbf{R}^{3}$. The edge map was derived 
from the segmented 3-D binary image of the nodule having property that it is larger near the nodule edges. The edge map was represented as

$$
g(\mathbf{x})=G_{\sigma} *|\nabla f(\mathbf{x})|
$$

where $G_{\sigma}$ is 3-D Gaussian function with the width $\sigma$. The edge map has the following three properties; (1) the gradient of an edge map $\nabla g(\mathbf{x})$ has vector toward the edges, which are normal to the edge, (2) these vectors have large magnitudes in the vicinity of the edge, and (3) in the homogeneous region $f(\mathbf{x})$ is nearly constant and $\nabla g(\mathbf{x})$ is nearly zero. Because of the first property, the normal direction of the nodule surface are approximated by the gradient of the edge map in the vicinity of the edges. Since this property is utilized to measure the relationship between nodule surface and surrounding structures, the first property is a desirable property. The capture range will be small due to the second property. The homogenous regions at the surrounding nodule will have zero. The last two properties are undesirable. The approach is to keep the desirable property of the gradients near the nodule edges, but to expand the gradient away from the edges into homogeneous regions of the nodule surrounding using a computational diffusion process. A vector field obtained by the computational diffusion process is denoted as gradient vector (GV) field. The GV field was defined as the vector field $\mathbf{h}(\mathbf{x}): \mathbf{R}^{3} \rightarrow \mathbf{R}^{3}$ that minimized the energy functional

$$
E=\int_{\mathbf{R}^{3}} \mu|\nabla \mathbf{h}|^{2}+|\nabla g|^{2}|\mathbf{h}-\nabla g|^{2} d \mathbf{x}
$$

where the gradient operator $\nabla$ is applied to each component of $\mathbf{h}$ separately. Minimizing Eq. (9) makes the vector field smooth when there is no data. When $|\nabla g|$ is small, the first term dominates the energy. On the other hand the second term dominates the energy as $|\nabla g|$ is large. The parameter $\mu$ is a regularization parameter controlling the tradeoff between the first term and the second term. Using the calculus of variations, it is found that the GV field must satisfy the Euler equation. We implemented the iteration technique presented by $\mathrm{Xu}$ [15] to solve the Eq. (9).

\subsection{Characterization of the Nodule Surrounding Based on Vector Fields}

Each voxel in the nodule surrounding was represented by the following six attributions; (1) the shape index value (denoted as SH), (2) the curvedness value (denoted as CV), (3) the CT density value (denoted as D), (4) the direction of maximum principal curvature (denoted as MPV), (5) the gradient vector (GV), and (6) the absolute value of inner product of MPV and GV (denoted as F). To select the central part of cylindrical or conic structures, we specified two threshold values $\mathrm{T}_{\mathrm{SH}}$ and $\mathrm{T}_{\mathrm{CV}}$ for $\mathrm{SH}$ and $\mathrm{CV}$, respectively. We then obtained the region consisting of voxels which satisfied with a condition $A$ of $\mathrm{SH} \geqq \mathrm{T}_{\mathrm{SH}}$ and $\mathrm{CV} \geqq \mathrm{T}_{\mathrm{CV}}$. This process 

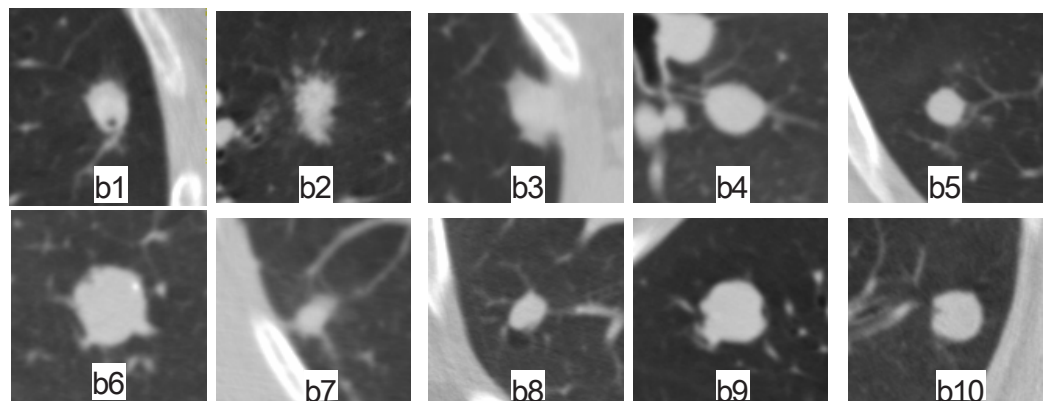

(a)
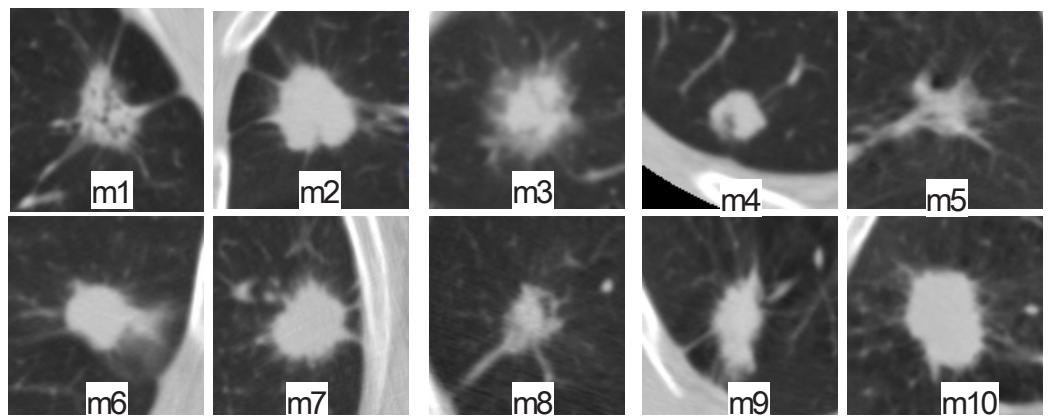

(b)

Fig. 1. Slice images of the ROIs of benign and malignant nodules. (a) Benign cases. (b) Malignant cases.

means that the vessel and pleura regions with cylindrical or conic structures are segmented and the central parts of these regions are selected because the curvedness value of the central part of cylindrical or conic structures become larger than that of the marginal part of them. We finally computed the ratio of the number of voxel concentrating on the nodule to the total number of voxel in the selected region. This ratio was represented as feature $F 1$. The feature $F 1$ was used as a measure of the ratio of vessels or pleura concentrating on the nodule

\section{Experimental Results}

The data set in this study included twenty 3-D thoracic images acquired from National Cancer Center Hospital East. Among the 20 cases, 10 contained malignant nodules, and 10 contained benign nodules. Whole malignant nodules were cytologically or histologically diagnosed. Fig. 1 presents slice images of the ROIs of benign and malignant nodules used here. In malignant cases, $\mathrm{m} 7$ and $\mathrm{m} 10$ were diagnosed as squamous cell carcinoma and others were diagnosed as adenocarcinoma. In benign cases, b1, b2, b3, b5, and b7 were diagnosed as inflammatory and others were diagnosed as tumor-like lesion. 


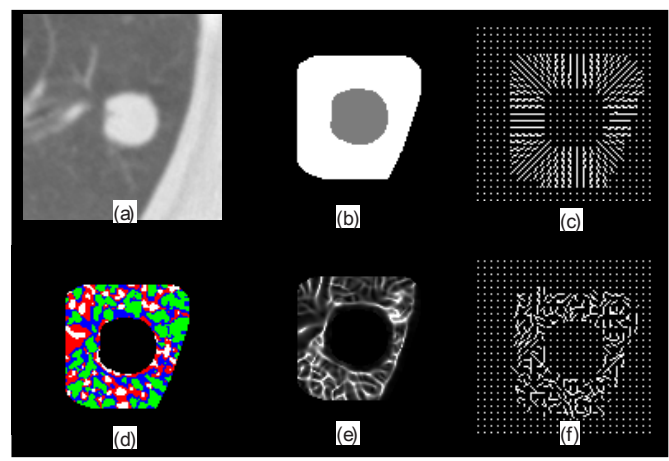

Fig. 2. Computation results of a benign case. (a) Slice image of the ROI. (b) Nodule surrounding region. (c) Gradient vector field. (d) Distribution of shape index. (e) Distribution of curvedness. (f) Vector field of the direction of the maximum principal curvature.

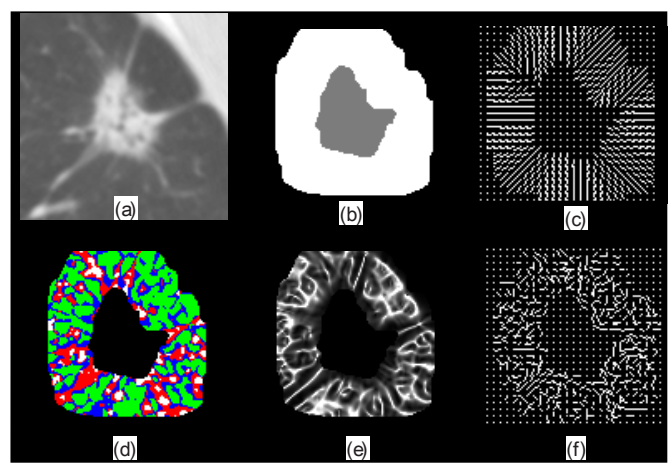

Fig. 3. Computation results of a malignant case. (a) Slice image of the ROI. (b) Nodule surrounding region. (c) Gradient vector field. (d) Distribution of shape index. (e) Distribution of curvedness. (f) Vector field of the direction of the maximum principal curvature.

Fig. 2 shows the distribution of the attributions on a slice of the ROI of a benign case. Fig.2 (a) shows the distribution of CT density value on the slice of the ROI and (b) presents the slice image of nodule surrounding colored white and the segmented nodule colored gray. Fig.2 (c) demonstrates the distribution GV field in the nodule surrounding. Fig. 2 (d), (e), and (f) demonstrate the distribution of the shape index value, the curvedeness, and the vector field of the maximum principal curvature in the slice of the nodule surrounding, respectively. In the shape index distribution, the defined color code corresponding to each surface type is as follows; peak surface is white, saddle ridge surface is red, saddle valley surface is blue, and pit surface is green. In the curvedness distribution the defined gray scale corresponding to the curvedness value is as follows; the value zero is black, the value lager than one is white, and the interval from 0 to 1 is represented by gray. In Fig.2 (c) and (f) the length of each vector was normalized to one. Fig. 3 shows the distribution of the attributions on a slice of the ROI of a malignant case. The figure arrangement is the same as that of Fig.2. In Fig.3 (a) it is observed that vessel and plural images are 
presented in the nodule surrounding. It is also observed that the cylindrical or conic structures such as vessel and plural image are represented as peak surface or saddle ridge surface types in Fig.2(d) and Fig.3 (d), and the center part of the structures have high curvature value in Fig.2 (e) and Fig.3 (e). In Fig.2 (f) and Fig. 3 (f) it is observed that directions of the maximum principal curvatures in the part corresponding to the cylindrical or conic structures are parallel to the axial directions of the structures.

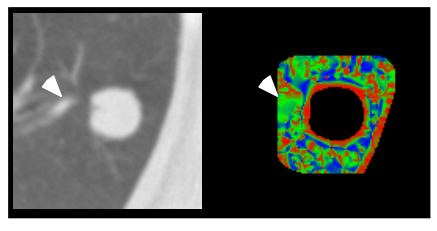

(a)

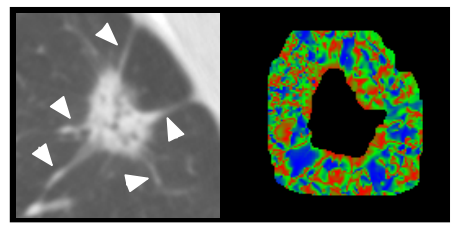

(b)

Fig. 4. Distribution of $F$ value of the nodule surrounding. (a) Benign case. (b) Malignant case. Left: Slice image of the ROI. Right: Distribution of $F$ value represented by a color code. The range of $F$ value is between 0 and 1 .

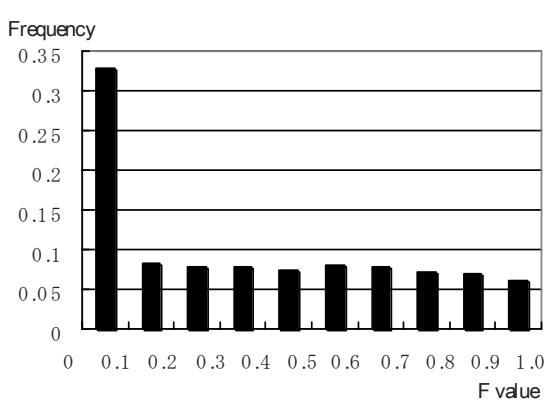

(a)

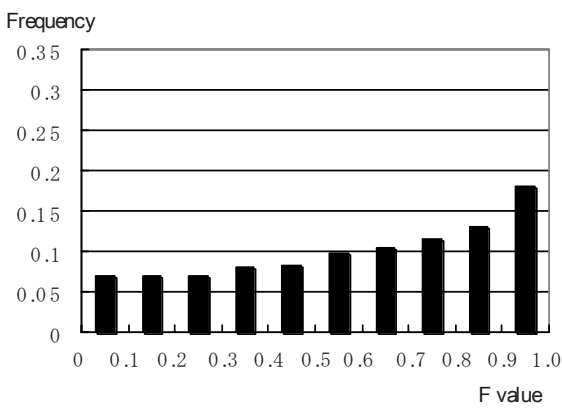

(b)

Fig. 5. Histogram of $F$ value. (a) Benign case. (b) Malignant case.

Fig. 4 shows the distribution of $F$ value of the nodule surrounding. The $F$ value is represented by a color code as shown in Fig.4. The color code is varied from red to blue as $F$ value becomes zero to one. The vessel region converging on the nodule and pleura region radiating from the nodule denoted by the arrows are colored blue. From these results it can be expect that the frequency of high $F$ value is depended on the existence of vessel or pleura converging on the nodule.

Fig. 5 shows a histogram of the $F$ value of the region constructed by a set of voxels which are satisfied with the condition $A$. In this experiment, the values of $\mathrm{T}_{\mathrm{SH}}$ and $\mathrm{T}_{\mathrm{CV}}$ were set to 0.5 and 0.9 , respectively. The frequency was normalized by the total number of voxels of the constructed region. Compared the distribution of the histogram of the benign case with that of the malignant case, it is observed that the voxels with higher value of $F$ occupy in the nodule surrounding. From these results it can be expect that the frequency of high $F$ value is depended on the existence of vessel or pleura converging on the nodule. 


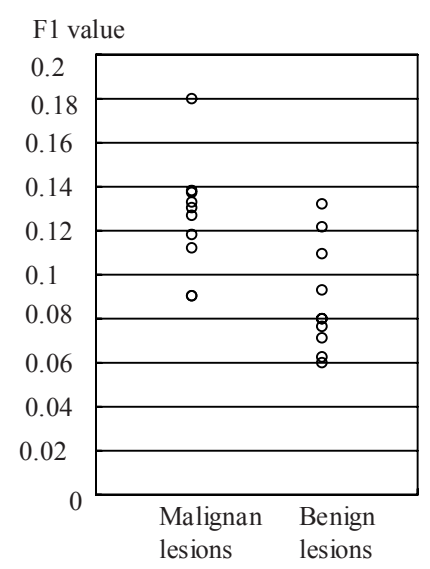

Fig. 6. Distribution of $F 1$ feature value of each pulmonary nodule shown in Fig. 1.

To analyze the statistical significance of the data, we divided the patients into two groups; patients with malignant and benign nodules. The feature $F 1$ value was given by the frequency of the range of $F$ value from 0.9 to 1.0. Fig. 6 shows the distributions of $F 1$ for two groups. The distributions of $F 1$ value were compared for the two groups based on the Wilcoxon rank sum test because the distribution of their variables might be nongaussian. Fig. 6 presents the distribution of $F 1$ value of each nodule. Based on the Wilcoxon rank sum test, the $F 1$ values of the malignant lesions were significantly higher than those of the benign lesions $(p<0.01)$. The benign cases diagnosed as tumor-like lesion were successfully distinguished from the malignant cases. While the distribution of the $F 1$ value of the benign cases diagnosed as inflammatory overlapped with that of the malignant cases. There is a benign lesion in which the vascular convergence and pleural retraction are observed in the nodule surrounding [1], [2]. Therefore, the potential of the feature F1 for nodule classification may be limited.

\section{Conclusion}

In this study, we have introduced the differential geometrical quantities for the structure analysis of nodule surrounding and evaluated the feasibility of differential geometry based vector fields in classifying malignancies from other lesions. While the number of studied nodules in our data set was too small to achieve a definitive conclusion, the preliminary result from this work was very encouraging, demonstrating the potential for vector field analysis used in the computerized characterization to discriminate benign from malignant pulmonary nodules.

We have demonstrated classification strategy of benign and malignant nodules based on the differential geometrical quantities in the nodule surrounding. The classification strategy was designed by an interactive analysis of the local intensity structures. Further work should therefore include the development of an automated method of the parameter adjustment such as the width of the Gaussian smoothing 
kernel. In addition, we will also investigate classification schemes considering the multidimensional feature space.

\section{References}

1. K.Mori, Y.Saitou, K.Tominaga, K.Yokoi, N.Miyazawa, A.Okuyama, M.Sasagawa, "Small nodular legions in the lung periphery: new approach to diagnosis with CT," Radiology, Vol.177, pp.843-849 (1990)

2. K. Kuriyama, R. Tateishi, O. Doi, K. Kodama, M. Tatsuta, M Matsuda, T. Mitani, Y. Narumi, M. Fujita, "CT-pathologic correlation in small peripheral lung cancers", AJR, Vol.149, pp.1139-1143 (1987)

3. T.Tozaki, Y.Kawata, N.Niki, H.Ohmatsu, R. Kakinuma, K.Eguchi, N. Moriyama, "Pulmonary organs analysis for differential diagnosis based on thoracic thin-section CT images", IEEE Trans. Nuclear Science, Vol.45, pp.3075-3082 (1998).

4. Y.Hirano, Y.Mekada, J.Hasegawa, J. Toriwaki, H.Ohmatsu, and K.Eguchi, "Quantification of vessels convergence in three-dimensional chest X-ray CT images with three-dimensional concentration index", Medical Imaging Technology, Vol.15, pp.228-236 (1997)

5. J. Williams, L. Wolff, "Analysis of the pulmonary vascular tree using differential geometry based vectored fields", Computer Vision and Image Understanding, Vol. 65, pp.226-236 (1997)

6. Y. Sato, S. Nakajima, N. Shiraga, H. Atsumi, S. Yoshida, T. Koller, G. Gerig, and R. Kikins,"Three-dimensionla multi-scale line filter for segmentation and visualization of curvlinear structures in medical images", Medical Image Analysis, Vol.2, pp.143-168 (1998)

7. Y.Kawata, N.Niki, H.Ohmatsu, R.Kakinuma, K.Eguchi, M.Kaneko, N.Moriyama, "Quantitative surface characterization of pulmonary nodules based on thin-section CT images", IEEE Trans. Nuclear Science, Vol. 45, pp.2132-2138 (1998)

8. Y. Kawata, N.Niki, H.Ohmatsu, M. Kusumoto, R. Kakinuma, K. Mori, K. Eguchi, M. Kaneko, N. Moriyama, "Classification of pulmonary nodules in thin-section CT images by using multi-scale curvature indexes", IEEE Int. Conf. on Image Processing, Vol.2, pp.197201, (1999)

9. Y. Kawata, N.Niki, H.Ohmatsu, "Curvature based internal structure analysis of pulmonary nodules using thoracic 3-D CT images", IEICE Trans., Vol.J-83-D-II, pp.209-218 (2000)

10.V.Caselles, R.Kimmel, G.Sapiro, and C.Sbert, "Minimal surfaces based object segmentation", IEEE Trans. Pattern Analysis Machine Intelligence, Vol.19, pp.394-398 (1997)

11. J.-P, Thirion and A. Gourdon, "Computing the differential characteristics of isointensity surfaces," Comput. Vision and Image Understanding, Vol.61, pp.190-202 (1995)

12. R. Deriche, "Recursively implementing the gaussian and its derivatives", INRIA Research Report, 1893 (1993)

13. J. J. Koenderink and A.J.V. Doorn, "Surface shape and curvature scales", Image and Vision Computing, Vol. 10, pp.557-565, (1992)

14. C.Dorai and A.K. Jain, " COSMOS-A representation scheme for 3D free-form objects", IEEE Trans. Pattern Analysis Machine Intelligence, Vol.19, pp.1115-1130 (1997)

15.C. Xu and J.L. Prince, "Snake, shape, and gradient vector flow", IEEE Trans. Image Processing, Vol. 7, pp.359-369 (1998) 